safety and efficacy of a higher dose of SAR97276A IM once $(0.5 \mathrm{mg} / \mathrm{kg})$ or twice $(0.25 \mathrm{mg} / \mathrm{kg})$ daily for 3 -days compared to artemether-lumefantrine in children $12-17$ years before age de-escalation to younger children (NCT01445938).

Results In the first study 113 patients received SAR97276A: 30 adults single-dose IV, 34 adults single-dose IM, 30 adults 3-day dose IM and 19 children 3-day dose IM. SAR97276A given as a single-dose to adult patients showed insufficient efficacy by IM route ( 20 cured of 34 ; and IV route (23/30 cured). The 3 -day treatment showed a sufficient level of efficacy when given IM to adults (27/30 cured) but not when given to children $7-17$ years (13/19 cured).

In the second study 20 patients were recruited and randomly assigned (2:2:1 ratio) to receive once-daily SAR97276A, twicedaily SAR97276A or artemether-lumefantrine. All patients receiving SAR97276A once-daily and 5/8 patients receiving SAR97276A twice-daily required rescue therapy. All patients in the control group were cured.

Conclusion Both studies were stopped due to lack of efficacy. SAR97276A given as monotherapy up to three days is not efficaciously curing malaria.

The studies were funded by Sanofi.

\title{
PA-021 SAFETY AND EFFICACY OF SAR97276A FOR TREATING MALARIA: TWO OPEN-LABEL MULTICENTER PHASE II CLINICAL STUDIES IN AFRICAN CHILDREN AND ADULTS
}

\author{
Jana Held, ${ }^{1,2,3}$ Christian Supan, ${ }^{1,2}$ Carmen L. Ospina Salazar, ${ }^{1,2}$ Halidou Tinto, ${ }^{4}$ Léa \\ Nadège Bonkian, ${ }^{4}$ Alain Nahum, ${ }^{5}$ Ali Sié, ${ }^{6}$ Salim Abdulla, ${ }^{7}$ Cathy Cantalloube, ${ }^{8}$ \\ Elhadj Djeriou, ${ }^{8}$ Marielle Bouyou-Akotet, ${ }^{9}$ Bernhards Ogutu, ${ }^{10}$ \\ Benjamin Mordmüller, ${ }^{1,2,3}$ Mohamadou Siribie, ${ }^{11}$ Sodiomon B. Sirima, ${ }^{11,12}$ Peter \\ G. Kremsner1,2,3. 'Institut für Tropenmedizin, Universitätsklinikum Tübingen, \\ Tübingen, Germany; ${ }^{2}$ Centre de Recherches Médicales de Lambaréné, Lambaréné, \\ Gabon; ${ }^{3}$ German Centre for Infection Research, partner site Tübingen, Germany; \\ ${ }^{4}$ Centre Muraz - IRSS, Bobo-Dioulasso, Burkina Faso; ${ }^{5}$ Centre de Recherche \\ Entomologique de Cotonou, Benin ; ${ }^{6}$ Centre de Recherche en Santé de Nouna, \\ Burkina Faso; ${ }^{7}$ Ifkara Health Research Center, Bagamoyo, Tanzania; ${ }^{8}$ Sanofi \\ Research and Development, France; ${ }^{9}$ Département de Parasitologie, Mycologie, \\ Médecine Tropicale, Université des Sciences de la Santé, Libreville, Gabon; ${ }^{10} \mathrm{KEMRI}$ \\ Walter Reed Project, Kisumu, Kenya; ${ }^{11}$ Groupe de Recherche Action en Santé, \\ Burkina Faso, ${ }^{12}$ Centre National de Recherche et de Formation sur le Paludisme, \\ Burkina Faso
}

\subsection{6/bmjgh-2016-000260.58}

Background SAR97276A, is a choline analogue inhibiting the phospholipid biosynthesis of Plasmodium falciparum. Treatment options for severe malaria are limited and SAR97276A represents a drug candidate for this indication.

Methods This is a report on two consecutive trials evaluating safety and efficacy of parenterally administered SAR97276A for the treatment of malaria.

The first study was a phase 2, multicenter, open-label study at six African hospitals (NCT00739206). At first adults with uncomplicated malaria were included receiving a single dose SAR97296A (IM: $0.18 \mathrm{mg} / \mathrm{kg}$ or IV: $0.14 \mathrm{mg} / \mathrm{kg}$ ) followed by repeated dosing with daily administration of the IM dose for three days in case of lack of efficacy of the single-dose regimen before age de-escalation.

The second study was a phase 2, multicenter, randomized, controlled open label study at five African hospitals assessing 